\title{
Efficacy of Single Burr Hole in Management of Chronic Subdural Hematoma
}

\author{
Ahmed M. Elshanawany*, Ahmed Elsayed Abokresha, Mohamed Mahmoud \\ Neurosurgery Department, Faculty of Medicine, Assiut University, Assiut, Egypt \\ Email: ^aelshanawany@ymail.com
}

How to cite this paper: Elshanawany, A.M., Abokresha, A.E. and Mahmoud, M. (2020) Efficacy of Single Burr Hole in Management of Chronic Subdural Hematoma. Open Journal of Modern Neurosurgery, 10, 81-87.

https://doi.org/10.4236/ojmn.2020.101009

Received: October 19, 2019

Accepted: December 2, 2019

Published: December 5, 2019

Copyright () 2020 by author(s) and Scientific Research Publishing Inc. This work is licensed under the Creative Commons Attribution International License (CC BY 4.0).

http://creativecommons.org/licenses/by/4.0/

\begin{abstract}
Background: Surgery of chronic subdural hematoma (CSDH) is a common practice of neurosurgeons. CSDH is considered a pathology of the elderly and usually many co-morbidities are present. CSDH carries high risk of mortality if not treated or not treated well. So, the minimal, safe and adequate surgical intervention is the bases to choose specific surgical maneuver. Aim of the Study: The efficacy of a single burr in evacuation and treatment of chronic subdural hematoma. Methods: This study was held in Assiut university hospitals, neurosurgical department. It is a prospective randomized controlled study. 113 patients were enrolled in this study in the period between March 2018 and June 2019. We included all cases of chronic subdural hematoma $(\mathrm{CSDH})$ and subacute subdural hematoma that were evacuated with single burr hole. Hematomas either, unilateral or bilateral were included. Septated hematomas were excluded. Patients' clinical characteristics as age, sex, clinical presentation, co-morbidities and patients outcome were recorded. Postoperative status as regards improvement of the neurological status, complications and recurrence of hematoma were recorded. Results: We had 113 patients operated via properly situated single burr hole in our study. 113 patients were included in this study, 79 males (69.9\%) and 34 females (30.1\%). Age of the patients ranges between 27 years old and 90 years old. Postoperative follow up was evaluated according to Glasgow outcome score (GOS). We had 98 patients with good recovery, 4 patients with moderate disability, 6 patients with severe disability and 5 patients died. Complications were in form of 3 patients with residual hematoma, 2 patients with tension pneumocephalus, 7 patients developed postoperative seizure, 5 cases developed cortical/parenchymal hematoma and 9 cases developed hematoma recurrence. Conclusion: Single, properly situated burr hole with previously described characters is an effective treatment option in cases of CSDH. It helps adequate, effective and safe removal of the pathology with limited maneuver.
\end{abstract}




\section{Keywords}

Chronic Subdural Hematoma, Single Burr Hole Evacuation, Complications

\section{Background}

Chronic subdural hematoma (CSDH) is defined as the presence of liquefied blood within a membrane in the cavity after 3 weeks or more of acute bleeding in the subdural space [1]. Its pathogenesis is a progressive process and if not treated, it may carry a great risk for severe morbidity and mortality [2]. Standard treatment of CSDH is surgical evacuation [3]. CSDH is considered a disease of the elderly and most patients have many comorbidities such as cardiac diseases, chronic antiplatelet therapy, hepatic and etc. These co-morbidities affect the prognosis and feasibility of surgical intervention [3]. The best surgical procedure for treatment of chronic subdural hematoma is that allow adequate drainage with short operative period and least invasive. The aim of this work is to evaluate the efficacy of a single burr in evacuation and treatment of chronic subdural hematoma.

\section{Methods}

This study was held in Assiut university hospitals, neurosurgical department. It is a prospective randomized controlled study. 113 patients were enrolled in this study in the period between March 2018 and June 2019. Those patients who were admitted in our department during the previous period in our department. We included all cases of chronic subdural hematoma (CSDH) that need surgical evacuation. Subacute subdural hematoma was included as they need the same treatment option. Hematomas either, unilateral or bilateral were included. Septated hematomas were excluded. Patients' clinical characteristics as age, sex, clinical presentation, co-morbidities and patients outcome were recorded.

Hematoma evacuation was performed through single burr hole. The burr hole was situated over the maximum thickness of hematoma. In cases when the hematoma thickness almost equal, burr hole is situated at the junction between anterior two thirds and posterior one third of the hematoma. Burr hole diameter is adjusted to be $20 \mathrm{~mm}$. To ensure adequate drainage and avoid air collection in subdural space, head position is designed to make burr hole in the uppermost point as much as we can. Subcutaneous drain around burr hole edge is left for 24 or 48 hours till stoppage of fluid drainage.

Postoperative status as regards improvement of the neurological status, residual hematoma and recurrence of hematoma were recorded. We defined patients with residual hematoma as that did not improve postoperatively and follow up CT revealed significant subdural collection with mass effect. We defined patients with hematoma recurrence as those developed reappearances of symptoms or re-accumulation of hematoma. Recorded clinical conditions on admission and on discharge were evaluated according to neurological grading system 
of Glasgow outcome scale (GOS) (Table 1) [4]. Average postoperative follow up periods range were three months.

\section{Results}

113 patients were included in this study, 79 Males (69.9\%) and 34 females (30.1\%). Age of the patients ranges between 27 years old and 90 years old (Table 2). The mean age was 69.37 . There were 63 patients were presented with disturbed conscious level. 39 patients were presented with right sided hemiparesis. Left hemiparesis was seen in 48 patients. Quadriparesis was seen in 19 patients. We observed manifestations of increased intracranial tension in 7 patients (Table 3).

Table 1. Glasgow outcome scale (GOS).

\begin{tabular}{cl}
\hline Item & \multicolumn{1}{c}{ Brief description } \\
\hline Dead & Patient died. \\
Vegetative state & $\begin{array}{l}\text { Condition of unawareness with only reflex response but periods of } \\
\text { spontaneous eye opening. }\end{array}$ \\
Severe disability & $\begin{array}{l}\text { Patient is conscious but dependent on others in daily activities, patient cannot } \\
\text { live independently and prevent work. }\end{array}$ \\
Moderate disability & $\begin{array}{l}\text { Patient is disabled but can live independently for daily activities. Disabilities } \\
\text { include various degrees of dysphasia, hemiparesis, as well as intellectual and } \\
\text { memory deficits. }\end{array}$ \\
Good recovery & $\begin{array}{l}\text { Resumption of normal activities despite presence of minor neurological } \\
\text { deficit or minor psychological impairment. }\end{array}$ \\
\hline
\end{tabular}

Table 2. Summary of patients' age group.

\begin{tabular}{cc}
\hline Age group & Number of patients \\
$20-29$ & 1 \\
$30-39$ & 1 \\
$40-49$ & 2 \\
$50-59$ & 6 \\
$60-69$ & 40 \\
$70-79$ & 42 \\
$80-89$ & 20 \\
90 and above & 1 \\
\hline
\end{tabular}

Table 3. Summary of patients' clinical presentation.

\begin{tabular}{cc}
\hline Clinical presentation & Number of patients \\
\hline Right hemiparesis & 39 patients \\
Left hemiparesis & 48 patients \\
Quadriparesis & 19 patients \\
Disturbed conscious level & 63 patients \\
Manifestations of increase intracranial tension & 7 patients
\end{tabular}


Right hemispheric hematomas were seen in 42 patients, left hemispheric hematomas were detected in 50 patients while bilateral hematomas were in 21 patients. The average hematoma size was $24 \mathrm{~mm}$. 81 patients $(71.7 \%)$ of our patients were having co-morbidities. 73 patients were on antiplatelet therapy for treatment of transient ischemic attacks or ischemic strokes. 51 patients were cardiac patients with different cardiac pathologies. 37 patients diagnosed with diabetes mellitus. 37 patients (32.7\%) were operated under local anesthesia and 76 patients $(67.3 \%)$ were operated under general anesthesia. The average real time since skin incision till skin closure is 23 minutes.

Postoperative follow up was evaluated according to Glasgow outcome score (GOS) and summarized in Table 4.3 cases $(2.7 \%)$ revealed no improvement in their neurological status, upon follow CT brain; residual hematoma was seen and re-tapping from the same burr hole. All the three cases improved after re-tapping. We had $2(1.8 \%)$ cases with tension pneumocephalus that needed re-opening and irrigation subdural space with saline from new frontal burr hole. 7 patients (6.2\%) experienced post evacuation seizures. All of them did not experienced seizures in the preoperative state. All of them were receiving prophylactic antiepileptic drugs. Those patients had emergency CT brain that revealed cortical or intraparenchymal hematoma in 5 patients. Upon follow up period 9 cases $(7.9 \%)$ developed hematoma recurrence. Hematoma recurrence occurred within 60 days of surgery. Those patients developed deterioration of their neurological status after initial improvement. Follow up CT brain revealed recurrence of the hematoma. We did not encounter any patient complicated with acute subdural hematoma. Complications were summarized in Table 5. We had 5 patients who died within the first week after surgery because of co-morbidities.

Table 4. Summary of outcome according to Glasgow outcome scale.

\begin{tabular}{cc}
\hline Markwalder grade & Number of patients \\
\hline Death & 5 \\
Vegetative state & 0 \\
Severe disability & 6 \\
Moderate disability & 4 \\
Good recovery & 98 \\
\hline
\end{tabular}

Table 5. Summary of postoperative complications.

\begin{tabular}{cc}
\hline Postoperative complication & Number of patients \\
\hline Residual hematoma & $3(2.7 \%)$ \\
Tension pneumocephalus & $2(1.8 \%)$ \\
Seizures & $7(6.2 \%)$ \\
Cortical or parenchymal hematoma & $5(4.4 \%)$ \\
Recurrence & $9(7.9 \%)$ \\
\hline
\end{tabular}




\section{Discussion}

Surgery of chronic subdural hematoma (CSDH) is a common practice of neurosurgeons. CSDH is considered a pathology of elderly and usually many co-morbidities are present. CSDH carries high risk of mortality if not treated or not treated well. Burr hole evacuation is considered the treatment of choice of CSDH [5] [6].

Evacuation of the hematoma through one burr hole is considered less minimal than evacuation through two burr holes. Also, it is better than using twist drill as it has better exposure and adequate hemostasis with the same minimal procedure. Rate of hematoma recurrence in literature is estimated between $5 \%$ and $30 \%$ [7] [8] [9] [10] [11]. In our study rate of recurrence was $7.9 \%$ of cases. In other studies that used single burr hole for hematoma evacuation, rate of recurrence was 2.98 [6]. Paul M. Brennan et al., in a found in their study that recurrence rate was $9 \%$ and there was no significant difference in recurrence rates between patients having one bur hole (10\%) or multiple bur holes (8\%) [12]. Nail, K. et al., reported in his randomized study over 254 patients that recurrence rates in single and double-burr-hole groups were $6.15 \%$ and $4.83 \%$ respectively, which was not statistically significant [13]. Many other studies revealed the same finding that there is no difference in rate of recurrence according to number of burr holes [14] [15] [16]. These findings are consistent with adequacy of single burr hole evacuation for optimum evacuation of CSDH.

Subdural tension pneumocephalus is defined as the presence of air within subdural space eliciting mass effect. This may follow CSCHs as it is commonly associated with cerebral atrophy and the associated increase in potential space in the subdural area [17]. In our study, $1.8 \%$ of cases developed tension pneumocephalus and required washing of subdural space with saline and reinsertion of subcutaneous drain. Hosni Salama series in evacuation of chronic subdural hematoma via single burr hole revealed no cases developed postoperative tension pneumocephalus [6]. In the literature, tension pneumocephalus ranged between 0 and $16 \%$ [18] [19]. Our results and others revealed efficacy of single burr hole as well as multiple burr holes in evacuation as regards occurrence of tension pneumocephalus.

In our series, post evacuation seizures were seen in 7 cases (6.2\%). 5 of them had cortical or intraparenchymal hematomas. Flores, G. et al., had percentage of 2.3 of their patients with postoperative seizures [20]. Chih-Wei, C. et al., reported an incidence of 5.4\% of postoperative seizure in their study [21]. In our series 5 patients had cortical and parenchymal hematomas. In the literature parenchymal hemorrhage is rare devastating complication that usually occurs in those with coagulopathy [22]. In our series only two of the five that developed post evacuation hemorrhage were with coagulopathy. The other 3 patients were with normal coagulation profile. The occurrence of hematomas in those patients could be explained on bases of forceful irrigation and rapid decompression of the brain after hematoma evacuation. To avoid occurrence of this serious com- 
plication, gradual drainage of the hematoma is recommended.

\section{Conclusion}

Single, properly situated burr hole with previously described characters is an effective treatment option in cases of CSDH. It helps adequate, effective and safe removal of the pathology with limited maneuver.

\section{Ethical Clearance}

This work fulfills all the ethical requirements and criteria.

\section{Conflicts of Interest}

The authors declare no conflicts of interest regarding the publication of this paper.

\section{References}

[1] Tsutsumi, K., Maeda, K., Iijima, A., Usui, M., Okada, Y. and Kirino, T. (1997) The Relationship of Preoperative Magnetic Resonance Imaging Findings and Closed System Drainage in the Recurrence of Chronic Subdural Hematoma. Journal of Neurosurgery, 87, 870-875. https://doi.org/10.3171/jns.1997.87.6.0870

[2] Atkinson, J.L., Lane, J.I. and Aksamit, A.J. (2003) MRI Depiction of Chronic Intradural (Subdural) Hematoma in Evolution. Journal of Magnetic Resonance Imaging, 17, 484-486. https://doi.org/10.1002/jmri.10275

[3] Reinges, M.H., Hasselberg, I., Rohde, V., Küker, W. and Gilsbach, J.M. (2000) Prospective Analysis of Bedside Percutaneous Subdural Tapping for the Treatment of Chronic Subdural Haematoma in Adults. Journal of Neurology, Neurosurgery, and Psychiatry, 69, 40-47. https://doi.org/10.1136/jnnp.69.1.40

[4] Wilson, J.T., Slieker, F.J., Legrand, V., Murray, G., Stocchetti, N. and Maas, A.I. (2007) Observer Variation in the Assessment of Outcome in Traumatic Brain Injury: Experience from a Multicenter, International Randomized Clinical Trial. Neurosurgery, 61, 123-128. https://doi.org/10.1227/01.neu.0000279732.21145.9e

[5] Drapkin, A.J. (1991) Chronic Subdural Hematoma: Pathophysiological Basis for Treatment. British Journal of Neurosurgery, 5, 467-473. https://doi.org/10.3109/02688699108998475

[6] Salama, H. (2019) Outcome of Single Burr Hole under Local Anesthesia in the Management of Chronic Subdural Hematoma. Egyptian Journal of Neurosurgery, 34, 8. https://doi.org/10.1186/s41984-019-0033-Z

[7] Santarius, T., Kirkpatrick, P.J., Ganesan, D., Chia, H.L., Jalloh, I., Smielewski, P., Richards, H.K., Marcus, H., Parker, R.A., Price, S.J., Kirollos, R.W., Pickard, J.D. and Hutchinson, P.J. (2009) Use of Drains versus No Drains after Burr-Hole Evacuation of Chronic Subdural Haematoma: A Randomised Controlled Trial. The Lancet, 374, 1067-1073. https://doi.org/10.1016/S0140-6736(09)61115-6

[8] Tsai, T.H., Lieu, A.S., Hwang, S.L., Huang, T.Y. and Hwang, Y.F. (2010) A Comparative Study of the Patients with Bilateral or Unilateral Chronic Subdural Hematoma: Precipitating Factors and Postoperative Outcomes. The Journal of Trauma, 68, 571-575. https://doi.org/10.1097/TA.0b013e3181a5f31c

[9] Ko, B.S., Lee, J.K., Seo, B.R., Moon, S.J., Kim, J.H. and Kim, S.H. (2008) Clinical Analysis of Risk Factors Related to Recurrent Chronic Subdural Hematoma. Journal 
of Korean Neurosurgical Society, 43, 11-15. https://doi.org/10.3340/jkns.2008.43.1.11

[10] Sikahall-Meneses, E., Salazar-Pérez, N. and Sandoval-Bonilla, B. (2008) Chronic Subdural Hematoma. Surgical Management in 100 Patients. Cirugia y Cirujanos, 76, 199-203.

[11] Chon, K.H., Lee, J.M., Koh, E.J. and Choi, H.Y. (2012) Independent Predictors for Recurrence of Chronic Subdural Hematoma. Acta Neurochirurgica (Wien), 154, 1541-1548. https://doi.org/10.1007/s00701-012-1399-9

[12] Brennan, P.M., Kolias, A.G., Joannides, A.J., Shapey, J., Marcus, H.J., et al. (2017) The Management and Outcome for Patients with Chronic Subdural Hematoma: A Prospective, Multicenter, Observational Cohort Study in the United Kingdom. Journal of Neurosurgery, 127, 732-739. https://doi.org/10.3171/2016.8.JNS16134

[13] Nayil, K., Altaf, R., Shoaib, Y., Wani, A., Laharwal, M. and Zahoor, A. (2014) Chronic Subdural Hematomas: Single or Double Burr Hole-Results of a Randomized Study. Turkish Neurosurgery, 24, 246-248.

[14] Xu, C., Chen, S., Yuan, L. and Jing, Y. (2016) Burr-Hole Irrigation with Closed-System Drainage for the Treatment of Chronic Subdural Hematoma: A Meta-Analysis. Neurologia Medico-Chirurgica (Tokyo), 56, 62-68. https://doi.org/10.2176/nmc.ra.2015-0013

[15] Kutty, S.A. and Johny, M. (2014) Chronic Subdural Hematoma: A Comparison of Recurrence Rates Following Burr-Hole Craniostomy with and without Drains. Turkish Neurosurgery, 24, 494-497.

[16] Zumofen, D., Regli, L., Levivier, M. and Krayenbühl, N. (2009) Chronic Subdural Hematomas Treated by Burr Hole Trepanation and a Subperiostal Drainage System. Neurosurgery, 64, 1116-1121. https://doi.org/10.1227/01.NEU.0000345633.45961.BB

[17] Miele, V.J., Sadrolhefazi, A. and Bailes, J.E. (2005) Influence of Head Position on the Effectiveness of Twist Drill Craniostomy for Chronic Subdural Hematoma. Surgical Neurology, 63, 420-423. https://doi.org/10.1016/j.surneu.2004.06.023

[18] Thapa, A. and Agrawal, B. (2009) Mount Fuji Sign in Tension Pneumocephalus. Indian Journal of Neurotrauma, 6, 161-162. https://doi.org/10.1016/S0973-0508(09)80016-6

[19] Ihab, Z. (2012) Pneumocephalus after Surgical Evacuation of Chronic Subdural Hematoma: Is It a Serious Complication? Asian Journal of Neurosurgery, 7, 66-74. https://doi.org/10.4103/1793-5482.98647

[20] Flores, G., Vicenty, J.C. and Pastrana, E.A. (2017) Post-Operative Seizures after Burr Hole Evacuation of Chronic Subdural Hematomas: Is Prophylactic Anti-Epileptic Medication Needed? Acta Neurochirurgica, 159, 2033-2036. https://doi.org/10.1007/s00701-017-3298-6

[21] Chen, C.-W., Kuo, J.-R. and Lin, H.-J. (2014) Early Post-Operative Seizures after Burr-Hole Drainage for Chronic Subdural Hematoma: Correlation with Brain CT Findings. Journal of Clinical Neuroscience, 11, 706-709.

https://doi.org/10.1016/j.jocn.2004.03.019

[22] Kim, J.K., Kim, S.W. and Kim, S.H. (2013) Intracerebral Hemorrhage Following Evacuation of a Chronic Subdural Hematoma. Journal of Korean Neurosurgical Society, 53, 108-111. https://doi.org/10.3340/jkns.2013.53.2.108 